\title{
Correction to: Correlation between the presence of herniation pit and femoroacetabular impingement: a systematic review and meta-analysis
}

\author{
Chul-Ho Kim ${ }^{1,2}$ · Sangwon $\mathrm{Han}^{3}$ • Cheol-Jung Yang ${ }^{2}$ · Jun Ho Kim ${ }^{2,4}$ (D)
}

Published online: 31 March 2020

(c) European Society of Sports Traumatology, Knee Surgery, Arthroscopy (ESSKA) 2020

\section{Correction to: \\ Knee Surgery, Sports Traumatology, Arthroscopy \\ https://doi.org/10.1007/s00167-020-05888-8}

Authors would like to correct the errors in figure 4 legend.

Figure 4 legend,

"Forest plots of the incidence of herniation pit (HP) in symptomatic hips (s) and asymptomatic..." should be corrected as "Forest plots of the incidence of herniation pit (HP) in symptomatic hips (a) and asymptomatic...".

The corrected Fig. 4 legend is given below:

The original article has been corrected.

The original article can be found online at https://doi.org/10.1007/ s00167-020-05888-8.

Jun Ho Kim

junojuno49@gmail.com

Chul-Ho Kim

oschulhokim@gmail.com

Sangwon Han

hswon87@naver.com

Cheol-Jung Yang

cjyangosdr@gmail.com

1 Department of Orthopedic Surgery, Gachon University Gil Medical Center, 21, Namdong-daero 774 beon-gil,

Namdong-gu, Incheon 21565, South Korea

2 Department of Orthopedic Surgery, Armed Forces Daejeon Hospital, 90, Jaun-ro, Yuseong-gu, Daejeon 34059,

South Korea

3 Department of Nuclear Medicine, Armed Forces Daejeon Hospital, 90, Jaun-ro, Yuseong-gu, Daejeon 34059, South Korea

4 Department of Orthopedic Surgery, Seoul Medical Center, 156, Sinnae-ro, Jungnang-gu, Seoul 02053, South Korea 


\begin{tabular}{|c|c|c|c|c|c|}
\hline 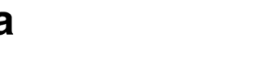 & FAl $(+$ & & FAl (- & & \\
\hline Study or Subgroup & Events & Total & Events & Total & Weight \\
\hline Ji et al. (2014) & 28 & 120 & 8 & 31 & $84.1 \%$ \\
\hline Kassarjian et al. (2005) & 2 & 39 & 0 & 3 & $8.0 \%$ \\
\hline Sullivan et al. (2013) & 1 & 17 & 0 & 49 & $7.9 \%$ \\
\hline Total $(95 \% \mathrm{Cl})$ & & 176 & & 83 & $100.0 \%$ \\
\hline Total events & 31 & & 8 & & \\
\hline Heterogeneity: $\operatorname{Tau}^{2}=0$ & $5 ; \mathrm{Chi}^{2}=2$ & $2.06, \mathrm{df}$ & $=2(P=c$ & 0.36); & $\left.\right|^{2}=3 \%$ \\
\hline Test for overall effect: $Z$ & $0.00(P=$ & n.s.) & & & \\
\hline
\end{tabular}

Odds Ratio Odds Ratio

-H, Random, $95 \%$ CI

$0.88[0.35,2.17]$

$0.47[0.02,11.77]$

$9.00[0.35,231.82]$

$1.00[0.40,2.51]$

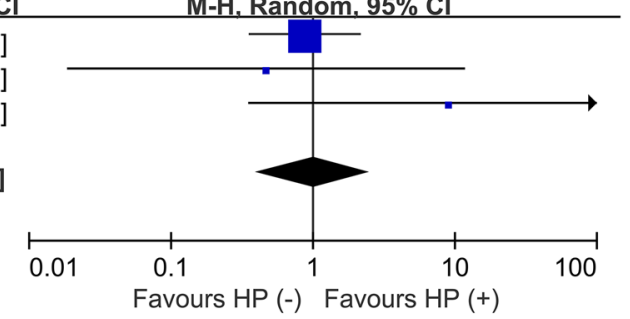

b

FAl (+) FAl (-) Odds Ratio

\section{Study or Subgroup} Events Total Events

Ji et al. (2014)

Lee et al. (2015)

Mineta et al. (2016)

381

nts Total Weight

Scheyerer et al. (2014)

$\begin{array}{rr}3 & 81 \\ 5 & 17\end{array}$

$2 \quad 70 \quad 0.4 \%$

$\begin{array}{lllll}114 & 773 & 50 & 405 & 9.6 \%\end{array}$

$\begin{array}{lllll}1001 & 3163 \quad 611 \quad 2765 \quad 89.1 \%\end{array}$

Total $(95 \% \mathrm{Cl})$

4034

$3408 \quad 100.0 \%$

Total events 1123 698

Heterogeneity: $\mathrm{Tau}^{2}=0.00 ; \mathrm{Chi}^{2}=2.25, \mathrm{df}=3(\mathrm{P}=0.52) ; \mathrm{I}^{2}=0 \%$

Test for overall effect: $Z=8.19(P<0.00001)$

Fig. 4 Forest plots of the incidence of herniation pit (HP) in symptomatic hips (a) and asymptomatic hips (b) between the groups with and without femoroacetabular impingement (FAI). The mean incidence of HP in symptomatic hips was similar between the two groups , 95\% C

$1.31[0.21,8.06]$

$1.58[0.52,4.79]$

$1.23[0.86,1.75]$

$1.63[1.45,1.83]$

$1.59[1.42,1.77]$

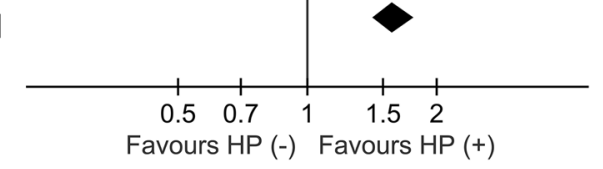

(OR 1.00; 95\% CI $0.40-2.51 ; P=1.00$ ). However, the incidence of HP in asymptomatic hips was higher in the group with FAI than the group without FAI (OR 1.59; 95\% CI 1.42-1.77; $P<0.0001$ )

Publisher's Note Springer Nature remains neutral with regard tojurisdictional claims in published maps and institutional affiliations. 